\title{
ANFÄNGE DES ANARCHISMUS. MARX` KRITIK DER HEGELSCHEN RECHTSPHILOSOPHIE UND SEIN POLITISCHER GEGENENTWURF
}

\author{
Steffen Wasko ${ }^{1}$
}

\begin{abstract}
Zusammenfassung:
Dieser Artikel führt die These aus, dass Marx‘ frühe Kritik an Hegel von 1843 ihn aufgrund seiner materialistischen Methode zu einer anarchistischen Position führt. Zwar stimmen Marx und Hegel durchaus in der Krisendiagnose ihre Zeit überein, allerdings trennen sich ihre Wege deutlich in Frage, wie diese Krise des Sozialen gelöst werden kann. Marx hat Hegels Analyse der Trennung zwischen der bürgerlichen Gesellschaft und dem modernen Staate viel zu verdanken, allerdings bleibt für ihn Hegels Ansatz der Vermittlung des Sozialen durch die Stände unhaltbar. Das Hauptproblem der Grundlinien der Philosophie des Rechts sieht Marx jedoch in Hegels idealistischer Methode and setzt daher der Logik seines Lehrers seine historischessentialistische Methode entgegen. Sichtlich beeinflusst von Feuerbachs Thesen, argumentiert Marx, dass der Staat und die bürgerliche Gesellschaft unmöglich miteinander vermittelbar sind und die moderne Form der Entfremdung und Unfreiheit eine notwendige bleiben muss. Da Staat und bürgerliche Gesellschaft unterschiedlichen Wesens sind, bleiben sie unvermittelbar und allein eine Revolution dieser beiden Formen des Sozialen kann die moderne Entfremdung aufheben. Die kursorischen Ausführungen Marx ' zu diesem unentfremdeten Zustand menschlicher Gesellschaft weisen des Weiteren deutliche Affinitäten zu klassischen anarchistischen Ansätzen auf.
\end{abstract}

Schlüsselwörter: Marx, Hegel, Anarchism, Critique.

\section{BEGINNINGS OF ANARCHISM. MARX CRITIQUE OF THE HEGELIAN PHILOSOPHY OF RIGHT AND HIS COUNTERDRAFT}

\begin{abstract}
:
This paper argues that Marx“ early citique of Hegel 1843 leads him, due to his materialisitic method, to a typical anarchist point of view. Despite the fact that both philosophers agree on a recent crisis of the social world, they disagree about the way this crisis could be solved. Marx follows Hegel in his analysis in which the social unity is divided by the differences between state and bourgeois society. But he criticises Hegel's attempt to mediate state and bourgeois society to a unity through the institution of the estates. Marx recognizes the main problem in Hegels Philosophy of Right is his method: He opposes his teacher's idealistic logic with his own historicalessentialist method. Obviously influenced by Feuerbachs Thesen, Marx argues, that state and bourgeois society could never be mediated, and that therefore modern humans live therefore by necessity unfree and alienated. Since bourgeois society and state hold on to different essence, only a revolution of both forms could end modern alienation. Furthermore, Marx ' cursory comments on the unalienated, free and stateless society show great affinity to classical anarchist approaches.
\end{abstract}

keywords: Marx, Hegel, Anarchism, Critique.

Einleitung

Hegel beendet seine 1806 in Jena gehaltene Vorlesung zur spekulativen Philosophie mit einer kritischen Zeitdiagnose:

1 2007-2015 Studium der Geographie, Geschichte und Philosophie in Freiburg und Patras. Seit 2015 Promotion zu Marx und Bakunin an der Albert-Ludwigs-Universität Freiburg (Philosophie). Steffen.Wasko@gmail.com. 


\begin{abstract}
Wir stehen in einer wichtigen Zeitepoche, einer Gärung, wo der Geist einen Ruck getan, über seine vorige Gestalt hinausgekommen ist und eine neue gewinnt. Die ganze Masse der bisherigen Vorstellungen, Begriffe, die Bande der Welt sind aufgelöst und fallen wie ein Traumbild in sich zusammen. Es bereitet sich ein neuer Hervorgang des Geistes. Die Philosophie hat vornämlich seine Erscheinung zu begrüßen und ihn anzuerkennen, während andere, ihm unmächtig widerstehend, am Vergangenen kleben und die meisten bewußtlos die Masse seines Erscheinens ausmachen. (zit. n. Hoffmeister 1936, 352.).
\end{abstract}

Der Beginn der Moderne, der mit der Französischen Revolution eingeleitet wurde, als Krise des Sozialen, als eine „Gärung“ zu fassen, ist nicht nur ein Grundtopos Hegels, ${ }^{2}$ sondern zugleich ein verbindendes Element zwischen diesem und Marx. Gleichwohl könnten ihre Antworten nicht unterschiedlicher sein. Während Hegel 1820 in seinen Grundlinien der Philosophie des Rechts aus diesem Krisenbewusstsein heraus den Versuch unternimmt, den vernünftigen Staat als eine das Soziale vermittelnde Instanz zu inaugurieren, so erteilt Marx dieser höchsten Manifestation des Sittlichen nicht nur eine deutliche Absage, sondern beschuldigt den Staat darüber hinaus, wesentlicher Akteur der Krise des Sozialen zu sein. Der Staat konstituiert die Entfremdung des Menschen von dem Menschen.

In Marx' Hegelkritik von 1843 steht dementsprechend die Staatskritik im Zentrum - doch zugleich geht es um mehr als Staatskritik. Marx' Hegelkritik verfährt vielmehr auf mehreren Ebenen. Einerseits versucht Marx die inneren Widersprüche der Hegelschen Staatsphilosophie aufzuzeigen indem er Hegel immanent kritisiert. Andererseits will Marx zeigen, dass die Hegelschen Kategorien nicht geeignet sind die Wirklichkeit darzustellen und zu verstehen. Gegen diesen Idealismus Hegels mobilisiert Marx eine materialistische Methode, welche ihrem eigenen Anspruch nach historisch fundiert ist und aus der schließlich sein anarchistisches Programm erwächst.

Im Folgenden möchte ich zeigen, wie aus Marx’ immanenter Hegelkritik zugleich eine eher äußere, materialistische Kritik des Staates und des Politischen überhaupt erwächst, die schließlich in dem mündet, was ich als Marx' frühen Anarchismus bezeichne. Nach der Darstellung der Affinitäten von Marx’ politischen Programm zum Anarchismus des 19. und

2 Joachim Ritter schreibt über die Zentralität der Französischen Revolution für Hegel: „Das Ereignis, um das sich bei Hegel alle Bestimmungen der Philosophie im Verhältnis zur Zeit, in Abwehr und Zugriff das Problem vorzeichnend, ist die Französische Revolution, und es gibt keine zweite Philosophie, die so sehr und bis in ihre innersten Antriebe hinein Philosophie der Revolution ist wie die Hegels" (Ritter 1957, 15).

\begin{tabular}{|c|c|c|c|c|}
\hline Rovista Dialeatus & Ano 9 & n. 18 & Outubro 2020 & p. $275-296$ \\
\hline
\end{tabular}


frühen 20. Jahrhundert unterziehe ich in einem letzten Schritt eine Kernkategorie dieser Konzeption einer Kritik - eine Kritik die für die Mehrheit anarchistische Theoriebildung des 19. Jahrhunderts gültig ist.

Hegels Vermittlung der bürgerlichen Gesellschaft und des Staates

Zunächst ist es allerdings unerlässlich knapp auf Hegels Darstellung der bürgerlichen Gesellschaft und des Staates im dritten Abschnitt des dritten Teils der Grundlinien der Philosophie des Rechts zu verlieren.

In diesem letzten Abschnitt widmet sich Hegel den Gliederungen des Sozialen, namentlich der Familie, der bürgerlichen Gesellschaft und schließlich dem Staat. Darin entwirft er ein Modell des Sozialen, welches zwar wesentlich vom Staat ausgeht, allerdings keine bloße Staatsphilosophie darstellt, sondern vielmehr eine organische Schichtung des Sozialen beschreibt, deren Anfangs- und Endpunkt der Staat bildet. Hegels Rechtsphilosophie kann sozusagen als der Versuch einer grundlegenden Vermittlung der theoretischen und empirischen Differenzen des Sozialen in der frühen Moderne angesehen werden. Die grundlegenden Erschütterungen des Sozialen in der Moderne, namentlich die Französische Revolution und der Siegeszug des Kapitalismus, sollen hier dem Anspruch nach wieder in einem organischen Ganzen zusammengefügt werden (vgl. Leopold 2009, 141).

Während also im bisherigen Gang der Rechtsphilosophie niedrigere Formen des Sozialen betrachtet wurden, wie die Familie und die bürgerliche Gesellschaft, so kommt Hegel nun im letzten Teil bei jener Form an, in welcher, „die Freyheit $\mathrm{zu}$ ihrem höchstem Recht kommt" (HGPR, 201) und in welcher zugleich alle Formen des sozialen und individuellem Lebens miteinander rational vermittelt werden - der Staat. Dementsprechend ist der Staat die höchste organische Einheit und rationalste Organisation des Sozialen. In Hegels Worten ist der Staat die „Wirklichkeit der sittlichen Idee“ (HGPR, 201), was in einer modernen Übersetzung bedeutet, dass der Staat die höchste Form ist, in der sich subjektives Bewusstsein in einer Einheit mit sozialen Institutionen objektiviert.

Wie bereits angedeutet, kommt dem Staat die Funktion zu, die unterschiedlichen Formen der Sittlichkeit, d.h. des Sozialen, organisch zu einen, ihre Gegensätze zu vermitteln und rational zu organisieren. Neben dem Staat selbst zählt Hegel eben die Familie und die bürgerliche Gesellschaft zu den elementaren Formen des Sozialen.

\begin{tabular}{|l|l|l|l|l|}
\hline Q Rovista Dialectus & Ano 9 & n. 18 & Outubro 2020 & p. 275-296 \\
\hline
\end{tabular}


Die Seinsweise innerhalb der Familie wird als Liebe charakterisiert, als „empfindende Einheit“" (HGPR, 144). Nachdem die Menschen aus der harmonisierenden Einheit der Familie scheiden, treten sie als nunmehr erwachsene, selbstständige Personen in die Sphäre des Systems der Bedürfnisse der bürgerlichen Gesellschaft ein. Hier gilt allerdings nicht jene Seinsweise der Liebe und der Einheit als soziales Prinzip, sondern gerade die einzelne, von sozialen Banden losgelöste Person mit ihren individuellen Bedürfnissen, sowie ihre Bezugnahme auf andere einzelne Personen. Die bürgerliche Gesellschaft hat gegenüber der sozialen Identität der Familie ihr soziales Prinzip wesentlich in einer relationalen Differenz einzelner Individuen, unter der Voraussetzung rechtlicher Gleichheit und individueller Freiheit - dem Individualismus (vgl. HGPR, 160).

Es ist ausdrücklich diese Sphäre des Privateigentums und Handels, die von Hegel und in seiner Nachfolge auch von Marx als die problematische betrachtet wird. Da die Grundlage der bürgerlichen Gesellschaft die allgemeine Gleichheit und Freiheit der Individuen ist und damit die Menschen allein als abstrakte, atomistische Individuen losgelöst von ihren konkreten Eigenschaften, d.h. jenseits ihrer sozialen Banden, Geltung besitzen, unterminiert sie die Konstitution einer Gesellschaft als Ganzes. Indes spaltet sie nicht allein formell, sondern konkret durch ihre Logik des Individualismus, des Privateigentums und der Konkurrenz aktiv die Gesellschaft. So fasst Hegel die Entwicklungslogik der bürgerlichen Gesellschaft wie folgt zusammen:

\footnotetext{
Wenn die bürgerliche Gesellschaft sich in ungehinderter Wirksamkeit befindet, so ist sie innerhalb ihrer selbst in fortschreitender Bevölkerung und Industrie begriffen. Durch die Verallgemeinerung des Zusammenhangs der Menschen durch ihre Bedürfnisse, und der Weisen, die Mittel für diese zu bereiten und herbeyzubringen, vermehrt sich die Anhäufung der Reichthümer [...] - auf der einen Seite, wie auf der anderen Seite die Vereinzelung und Beschränktheit der besondern Arbeit und die Abhängigkeit und Noth der an diese Arbeit gebundenen Classe, womit die Unfähigkeit der Empfindung und des Genusses der weitern Freyheiten und besonders der geistigen Vortheile der bürgerlichen Gesellschaft zusammenhängt. (HGPR, 193)
}

Die weitgehende Exklusion, Verarmung und Entfremdung eines Teils der Bevölkerung ist somit nach Hegel strukturelle Folge des Prinzips der bürgerlichen Gesellschaft. Hinzu kommt, dass die Frage, wer was arbeitet, also die Frage welche funktionale Teilnahme der einzelnen

\begin{tabular}{|c|c|c|c|c|}
\hline Rovita Didectus & Ano 9 & n. 18 & Outubro 2020 & p. $275-296$ \\
\hline
\end{tabular}


Person in der bürgerlichen Gesellschaft zukommt, vor allem durch die Zufälligkeit der jeweiligen Umstände bedingt ist (vgl. HGPR, 169).

Soll die Gesellschaft als Ganze einen organischen Zusammenhang bilden, so muss Hegel zeigen können, dass der Staat die Pathologien der bürgerlichen Gesellschaft dialektisch aufheben kann. Anders zerfällt die Sittlichkeit in verschiedene widerstreitende Subsysteme des Sozialen - und dies widerspräche Hegels Logik. Er ist allerdings überzeugt, dass die Vermittlung von Besonderen - die Einzelperson der bürgerlichen Gesellschaft - und Allgemeinen - der Mensch als sittliches Wesen - durch den Staat geleistet werden kann.

Hegels wichtigster Kandidat für die Vermittlung zwischen bürgerlicher Gesellschaft und dem Staat sind die Stände. Sie werden der gesetzgebenden Gewalt des Staates zugesprochen, nehmen aber bloß eine beratende Funktion ein (vgl. HGPR, 247f.). Die Stände selbst teilen sich wiederum in zwei Sektionen aus Teilen des Adels einerseits und Vertretern der Korporationen aus der bürgerlichen Gesellschaft andererseits (vgl. HGPR, 250-254). Hegel lehnt jegliches (basis-)demokratische Element bewusst ab, da damit ,alle vernünftige Form in de[m] Staatsorganismus“ (HGPR , 254) unterminiert würde und darüber hinaus durch allgemeine Wahlen ,,jedem der Weg offen [ist] auch sein subjectives Meynen über das Allgemeine zu äußern und geltend zu machen.“ (ebd.) Indem sich die Stände hierarchisch aus dem Adel und den Vertretern der Korporationen - d.h. der Handwerkerzünfte, Unternehmen und Kaufmannsgilden - zusammensetzen, entsprechen sie den jeweiligen sittlichen Sphären, die sie vertreten. Diesen Vertretern spricht Hegel die Kompetenzen zu, im Sinne ihre Abordnungen und mit Blick auf das soziale Allgemeine, welches der Staat vertritt, politisch zu handeln.

Gerade bei den Ständen aus der bürgerlichen Gesellschaft geht es Hegel darum, dass sich diese als Vertreter der Ordnung der bürgerlichen Gesellschaft, den Korporationen, konstituieren. Dadurch sei gewährleistet, dass eben nicht jener individualistische, zufällige, sondern der vernünftig sittliche Teil dieser sozialen Sphäre politische Macht erhält. Als vermittelnde Vertreter stehen die Stände ,zwischen der Regierung einerseits, und dem in besonderen Sphären und Individuen aufgelösten Volke andererseits.“ (HGPR , 250) Die Stände bilden die Mitte zwischen diesen beiden Sphären und haben damit die Bestimmung sowohl die „Gesinnung des Staates und der Regierung“, als auch die „Interessen der besonderen Kreise und der Einzelnen" zugleich zu vertreten. (HGPR, 250) Als Abordnungen der bürgerlichen Gesellschaft stehen sie daher nicht im einfachen Gegensatz zum Staat und

\begin{tabular}{|l|l|l|l|l|}
\hline Q Rovista Dialectus & Ano 9 & n. 18 & Outubro 2020 & p. 275-296 \\
\hline
\end{tabular}


der Regierung, sondern aus einer holistischen, organischen Perspektive „beweist sich das ständische Element nur durch die Function der Vermittlung.“ (HGPR, 251) Damit sei Hegel zufolge der Gegensatz zwischen Staat und bürgerlicher Gesellschaft zu „einem Schein herabgesetzt." (ebd.)

Mit diesem Modell einer politischen Ständegesellschaft richtet sich Hegel gegen die liberale Theorie, in der er die Gefahr von zwei Extrema erblickt. Zum einen bestünde die Gefahr „der Auflösung der bürgerlichen Gesellschaft in eine ,Menge von Individuen““, ein Manko, dass Hegel besonders in der Demokratie sieht. Zum anderen bestünde die Gefahr einer Formalisierung des politischen Staats (vgl. HGPR, 254f.). Hegel nimmt hingegen eine Position zwischen diesen beiden Extrema ein, die im Gegensatz zum Liberalismus und der Demokratie eine ,feste und berechtigte Grundlage“ (HGPR , 252) besitzt. Die Demokratie, welche allen Individuen der bürgerlichen Gesellschaft gleichsam die politische Betätigung ermöglicht, lehnt Hegel mit dem Verweis ab, dass damit bloß der abstrakten Vorstellung Rechnung getragen wird, Mitglied des Staats zu sein. Damit setze man „ohne alle vernünftige Form" (HGPR , 254) das abstrakte subjektive Element unvermittelt in den Staat. Denn der Staat, so führt Hegel aus, ,ist aber wesentlich eine Organisation [...] und in ihm soll sich kein Moment als eine unorganische Menge zeigen.“ (HGPR, 252)

Zentral für seine Kritik an der Demokratie ist Hegels Verständnis von Repräsentation, welches er aus dem Standpunkt der organischen Totalität gewinnt und sich grundsätzlich von jeglichem liberalen Paradigma unterscheidet. Gegen die liberale Auffassung von Repräsentation als Vertretung von Einzelnen schreibt Hegel:

\footnotetext{
Wenn die Abgeordneten als Repräsentanten betrachtet werden, so hat diß einen organischen vernünftigen Sinn nur dann, daß sie nicht Repräsentanten als von Einzelnen, von einer Menge seyn, sondern Repräsentanten einer wesentlichen Sphäre der Gesellschaft, Repräsentanten ihrer großen Interesse. (HGPR, 257)
}

Die Repräsentation in Form der Stände ermögliche schließlich die Einheit von Staat und bürgerlicher Gesellschaft in der Differenz.

Marx' immanente Kritik

Marx' immanente Kritik an Hegels Vermittlung von Staat und bürgerlicher Gesellschaft ist eine vielschichtige. Im Folgenden werde ich allerdings den Fokus auf die Kritik der Vermittlung legen. Aus einer historischen Perspektive wendet Marx zunächst ein, dass die

\begin{tabular}{|l|l|l|l|l|}
\hline Q Rovista Dialectus & Ano 9 & n. 18 & Outubro 2020 & p. 275-296 \\
\hline
\end{tabular}


Institution, welche Hegel als einen Kandidaten der Vermittlung einführt, die Ständeversammlung, völlig veraltet sei und bereits von der Französischen Revolution überholt wurde. Dementsprechend legt Marx in einem kurzen historischen Abriss die Entwicklung der Stände vom Mittelalter (als sie noch eine rein politische Funktion ausübten) über ihre Transformation in der absoluten Monarchie zu sozialen Ständen (in denen aber noch stets eine politische Funktion hegemonial blieb) dar. Schließlich entwickelten sie ihre gegenwärtige, rein soziale Form im Zuge der Französischen Revolution (vgl. MEW 1, 283f.).

Hegel hingegen nimmt eine rein begriffsgenetische Perspektive ein, wonach die Stände ein subjektives Moment im Hinblick auf das Allgemeine darstellen (vgl. HGPR, 250). Indem nun Hegel einerseits die Stände als Momente der bürgerlichen Gesellschaft begreift, diese wiederum als Sphäre des Eigeninteresses und dezidiert unpolitisch fasst, verwundert es Marx, wie die Stände nun innerhalb der staatlichen Ordnung eine politische Funktion ausüben können - Hegel hatte zuvor die Stände als Privatstand bezeichnet. Wesentlich seien sie eben gegen den Staat als Sphäre des Allgemeinwohls gerichtet und daher notwendig unpolitisch, d.h. nicht auf ein Allgemeines abzielend, sondern vielmehr auf ein jeweiliges Partikulares. Wie auch Hegel selbst ausführlich schildert, besteht die Seinsweise der bürgerlichen Gesellschaft im Prinzip des Individualismus, das eine bloß formale Allgemeinheit herstellt, die sich wiederum strukturell durch soziale Zufälligkeiten (der sozialen Stellung) und Extrema (von Reichtum und Armut) charakterisiert. Indem aber nun Hegel ihnen trotz ihres wesenhaften Individualismus eine politische Funktion zuschreibt, führt er Marx zufolge eine „Transsubstantiation“ (MEW 1, 280) durch, eine Wesensverwandlung. So folgert Marx, dass durch die Politisierung des Stands innerhalb der Ständeversammlung ,sich die bürgerliche Gesellschaft völlig von sich als bürgerlicher Gesellschaft, als Privatstand“ lossagt, damit eine „Partie seines Wesens geltend“ macht, die schließlich „mit der wirklichen bürgerlichen Existenz seines Wesens nicht nur keine Gemeinschaft hat, sondern ihr direkt gegenübersteht.“ (MEW 1, 280f.) Marx’ zweiter Einwand ist - nach dem historischen - ein essentialistischer, in dem Sinne, dass die Stände wesensmäßig unmöglich eine vermittelnde Funktion einnehmen können, da sie sonst sich selbst untergraben würden, also nicht mehr das wären was sie sind: Moment der bürgerlichen Gesellschaft. Damit habe Hegel eben nicht die Vermittlung der Sphären Staat - bürgerliche Gesellschaft geleistet, sondern vielmehr versucht ihre Trennung, die er zuvor als Widerspruch begreift, vertuscht.

\begin{tabular}{|c|c|c|c|c|}
\hline Rovista Dialeatus & Ano 9 & n. 18 & Outubro 2020 & p. $275-296$ \\
\hline
\end{tabular}


Marx teilt dementsprechend Hegels Analyse und Kritik der bürgerlichen Gesellschaft, widerspricht aber aus einer historischen und essentialistischen Perspektive vehement einer Vermittlung des Individualismus mit dem sittlichen Allgemeinen durch den Staat. Schließlich vermittelt die Ständeversammlung nicht die Sphären des privaten und des öffentlichen, sondern das „politisch-ständische Element“ ist „eben nichts anders [...] als der faktische Ausdruck des wirklichen Verhältnisses von Staat und bürgerlicher Gesellschaft, ihre Trennung.“ (MEW 1, 279) Diese Form der Kritik, welche von den grundsätzlich gegensätzlichen Wesen ausgeht, möchte ich als empirisch-essentialistischen Kritik bezeichnen. ${ }^{3}$ Sie ist zugleich eine immanente Kritik an Hegel, da sie an Hegels wesenhaften und logischen Unterscheidungen anknüpft.

Marx’ materialistischer Ansatz

Hegels großangelegte Vermittlung verfängt sich Marx zufolge in selbstverschuldeten Aporien, die sich einerseits aus Hegels Analyse und Kritik der bürgerlichen Gesellschaft und andererseits aus seinem idealistischen Logozentrismus ergeben.

Marx könnte nun den Weg gehen, in linkshegelianischer Manier einen besseren Versuch der Vermittlung zwischen Staat und bürgerlicher Gesellschaft auszuarbeiten, wie es beispielsweise sein Mentor Bruno Bauer versucht hat. ${ }^{4}$ Jedoch wird die Abkehr von Bauer 1843 deutlich, da sich Marx durch die immanente Kritik an Hegel in Verbund mit seiner Feuerbach-Lektüre, ${ }^{5}$ sowie seiner ausführlichen Beschäftigung mit der zeitgenössischen Geschichtsschreibung ${ }^{6}$ vielmehr einer materialistischen Methodik zuwendet und damit zu einer radikalen Kritik der politischen Form „Staat“ an sich gezwungen sieht. ${ }^{7}$ Die hier vertretene These lautet dementsprechend, dass die immanente philosophische Kritik der Hegelschen Philosophie des Staates und der Gesellschaft Marx’ Anarchismus gebiert.

3 Von besonderer Bedeutung für diesen Kritik-Modus ist das reichhaltige historische Material, das sich Marx während seiner Zeit in Kreuznach im Sommer 1843 angeeignet hat. Er studierte ungefähr 25 Jahrhunderte Weltgeschichte, dabei vor allem die jüngere Geschichte Frankreichs und im Besonderen der Französischen Revolution. Aber auch die Geschichte Englands und der USA sowie des Antiken Griechenlands, Venedigs, Polens und Schwedens. Vgl. MEGA IV/2. Dieses reiche Material erlaubt ihm Hegels Philosophie an der Geschichtsschreibung selbst zu prüfen.

$4 \quad$ Wie beispielsweise in Die Judenfrage, Braunschweig 1843.

5 Marx bezieht sich vor allem auf Vorläufige Thesen zur Reformation der Philosophie, wie er in einem Brief an Ruge anmerkt (vgl. MEGA III/1, 45).

6 Vgl. Marx' Exzerpte zur Geschichte verschiedener Staaten: MEGA IV/2; sowie Rubel 1968, 17f.

7 Zu der Beschäftigung Marx’ mit dem französischen Kommunismus/ Sozialismus vgl. MEGA IV/2.

\begin{tabular}{|l|l|l|l|l|}
\hline Rovita Dialectus & Ano 9 & n. 18 & Outubro 2020 & p. 275-296 \\
\hline
\end{tabular}


Im Gegensatz zu seiner Zeit als Journalist hat sich Marx ab August 1843 vom Republikanismus abgewendet und es wäre daher weit gefehlt den Marx von 1843 einen Demokraten zu nennen. ${ }^{8}$ Demgegenüber stellt das Programm Marx', welches er in der Kritik der Hegelschen Rechtsphilosophie unter dem Begriff der „wahren Demokratie“ fasst, einen anarchistischen Entwurf dar. ${ }^{9}$. Dieser Übergang vom Demokraten zum Anarchisten durch die Philosophie Feuerbachs, d.h. der Materialismus des Gattungswesens, ermöglicht. ${ }^{10}$

Dies wird in der Kritik der Hegelschen Rechtsphilosophie an vielen Stellen nur zu deutlich. In derselben Weise wie Feuerbach das religiöse Wesen in das menschliche auflöste, transferiert Marx Hegels idealistische Kategorien des Sittlichen in materialistische Kategorien des Politischen. ${ }^{11}$ Während Hegel den Staat als Anfangs- und Endpunkt setzt, welcher souverän über dem Volk stehe, so dreht Marx die Entwicklungslogik als auch das Verhältnis um: „Als wäre nicht das Volk der wirkliche Staat. Der Staat ist ein Abstractum. Das Volk allein ist das Concretum.“ (MEW 1, 229) Nicht vom Staat ist also auszugehen, sondern vielmehr von dem ihm zugrundeliegenden Subjekt, dem Volk. Nicht der Geist teilt sich in die Sphären der bürgerlichen Gesellschaft und der Familie, konstituiert schlussendlich den Staat und fügt die Menschen in ein organisches sittliches Ganzes, sondern der Staat ist vielmehr ein Moment des substanziellen Volkslebens. Marx schreibt:

\footnotetext{
Werden z.B. bei der Entwicklung von Familie, bürgerliche Gesellschaft, Staat etc., diese socialen Existentialweisen des Menschen als Verwirklichung, Verobjectivirung seines Wesens betrachtet, so erscheinen Familie etc. als einem Subjekt inhärente Qualitäten. Der Mensch bleibt immer das Wesen aller dieser Wesen, aber diese Wesen erscheinen auch als seine wirkliche Allgemeinheit, daher auch als das Gemeinsame. (MEW 1,241)
}

8 Marx selbst verweist auf die Abstraktheit und Unzulänglichkeit der liberalen Demokratieauffassung, vgl. MEW 1, 232.

9 Die Frage von immanenter und externer Kritik in dieser Schrift ist in der Forschung durchaus umstritten. Avineri bspw. spricht von einer rein immanenten Kritik (vgl. Avineri 1969, 12), während Abensour die externen Elemente der Kritik stark macht (vgl. Abensour 2012, 177-182).

10 Zwar teilt Marx weitgehend Feuerbachs Hegelkritik, allerdings beziehe sich Feuerbach Marx zufolge „zu sehr auf die Natur und zu wenig auf die Politik.“ (MEGA III/1, S.45.) Diesem Versäumnis kommt Marx in seiner Hegelkritik wiederum nach, wie im Folgenden ausgeführt wird (Zur Rolle Feuerbachs bei Marx' Hegelkritk vgl. Avineri 1969, 10-12). In diesem Sinne darf der Einfluss der staatstheoretischen Schriften von Machiavelli (Vom Staate), Rousseaus (Du contrat social), sowie Montesquieus (De l'esprit des loix) auf die Hegelkritik Marx' nicht unterschätzt werden. Zu Machiavellis Einfluss auf Marx vgl. Abensour 2012.

11 Bereits hier, 1843, beginnt jener bekannte Prozess, in dem Marx Hegels Philosophie ,vom Kopf auf die Füße“" stellt (vgl. Loick 2010, 155).

\begin{tabular}{|l|l|l|l|l|}
\hline Gonista Dialectus & Ano 9 & n. 18 & Outubro 2020 & p. 275-296 \\
\hline
\end{tabular}


Während für Hegel das Volk eine bloße „formlose Masse“ ist, in der die „Vielen als Einzelne“ (HGPR, 252) gerade kein organisches Ganzes ausbilden können, so dreht Marx auch hier wieder den Zusammenhang um: Das Volk ist das zugrundeliegende Subjekt, welches sich in besondere Momente verobjektiviert. Das Volk ist „der wirkliche Träger der Verfassung“ (MEW 1, 259) und eben der „wirkliche Staat“ (MEW 1, 229). Hegel meint aber „Alle“ als die Einzelnen, welche erst vernünftig organisiert werden müssen. Dagegen versteht Marx das Volk vor dem Hintergrund des Gattungswesens als „Alle“ im Sinne einer wirklichen, konkreten Einheit, als ein Kollektivsingular (vgl. Theunissen 1978, 480-482). Das Volk ist nicht formlose Masse, sondern vielmehr formgebende Substanz. ${ }^{12}$

Indem Marx die typische Inversion von Subjekt und Prädikat konsequent an Hegels Rechtsphilosophie durchdekliniert, damit Hegels idealistische Entwicklungslogik dekonstruiert, eröffnet er schlussendlich den Raum des Politischen. Während Hegel das Soziale wesentlich als vernünftige Wirklichkeit der Idee der Sittlichkeit fasst, so stellt sich für Marx aus der Feuerbachschen Perspektive das Soziale grundlegend als Zusammenhang der verschiedenen sozialen Wesenslogiken des Gattungswesens Mensch dar. Das Gattungswesens wiederum ergänzt Marx um eine eminent politische Perspektive, so dass das sozialphilosophische Fundament nunmehr um das politische Subjekt-Objekt des Volkes bereichert wird.

Gleichwohl bleiben trotz aller Kritik die tiefen Einsichten Hegels in die Strukturen des Sozialen der unhintergehbare Ausgangspunkt für Marx. ${ }^{13}$ Zwar führt ihn sein empirischessentialistischer Kritik-Modus zu einer Ablehnung des apriori Charakter der Kategorien Hegels und letztendlich zum Gattungswesen, bzw. Volk als Grundkategorie des Sozialen. Jedoch sind damit keineswegs jene höchstproblematischen Entfremdungsphänomene gelöst, welche Hegel in der Rechtsphilosophie aufreißt: Zum einen das Prinzip der bürgerlichen Gesellschaft, der Individualismus, und zum anderen das Auseinanderklaffen von bürgerlicher Gesellschaft und Staat. Auch wenn Hegel und Marx von unterschiedlichen politischen Standpunkten mit unterschiedlichen Grundkategorien argumentieren, so treffen sie sich in der

12 Eine ähnliche Kritik an Hegel findet sich schon 1839 bei Ruge, dessen Einfluss für Marx’ politischen Werdegang entscheidend war (vgl. Lindner 2013, 74-76). übergeordnete Instanz gegenüber der bürgerlichen Gesellschaft nicht einfach um, sondern fasst beide Sphären vielmehr als gleichberechtigt und entgegengesetzt. Zur älteren Forschung vgl. Althusser 1968; Hyppolite 1969, 112. Dagegen vgl. Lindner 2013, 84-8; Loick 2010, 156-157; Leopold 2009, 70-74.

\begin{tabular}{|c|c|c|c|c|}
\hline Q Rovista Oialectus & Ano 9 & n. 18 & Outubro 2020 & p. $275-296$ \\
\hline
\end{tabular}


Grundannahme, dass das Soziale eine Totalität bildet, die ferner eine Einheit sein soll. Marx' Argument gegen Hegels Vermittlung von bürgerlicher Gesellschaft und Staat ist ja gerade, dass diese Vermittlung aufgrund ihrer jeweiligen eigenständigen Wesenslogiken scheitern muss und die vorgängige Entfremdung dieser beiden sozialen Sphären schließlich unaufgelöst bleibt. Beide stimmen also sowohl in der Feststellung der sozialen Entfremdung wie auch in der Notwendigkeit ihrer Überwindung überein. Jedoch widerspricht Marx Hegel darin, dass die zeitgenössische moderne soziale Welt diese Bedingungen der Überwindung schon bereitstellen würde. Nicht also in seinem Anspruch kritisiert Marx Hegel, sondern vielmehr, dass er diesen schon mit der Überwindung der Entfremdung selbst verwechselt habe (vgl. Leopold 2009, 75f.).

Marx beschreibt die wesenslogische Trennung als eine Differenz von dem was er Inhalt des Staates, d.h. der rechtliche Rahmen in dem sich die bürgerliche Gesellschaft entfaltet, und der jeweiligen Staatsform, d.h. das jeweilige politische System, wie Monarchie, Republik, etc. ${ }^{14}$ Die Trennung von Inhalt und Form des Staates fasst Marx zugleich unter den Begriffen vom „materielle[n] Staat" ${ }^{\star 15}$ und dem ,,abstrakte[n] Staat ${ }^{\text {"16 }}$. Die materielle, bzw. inhaltliche Sphäre des Staates manifestiert sich vor allem in der bürgerlichen Gesellschaft, da sich hier konkret die materielle Produktion des menschlichen Lebens - das System der Bedürfnisse, wie Hegel sagt - abspielt. Der inhaltliche Staat, also das politische System, hat zwar die Wahrung des Allgemeinwohls zur Aufgabe, vermag allerdings nicht wesentlich in die materielle Produktion einzugreifen. Im Zusammenhang dieser Trennung des abstrakten Staates von der bürgerlichen Gesellschaft spielt das jeweilige politische System allerdings keine Rolle. Nach Marx ist es also einerlei, ob der abstrakte Staat eine Monarchie, oder eine Republik sei - beides seien nur verschiedene Erscheinungsformen der grundlegenden Form des abstrakten Staates (vgl. MEW $1,232)$

Demgegenüber stellt Marx auf Basis seiner historischen Forschung fest, dass diese Trennung Resultat der konkreten historischen politischen Prozesse ist. Im Mittelalter hingegen bildeten Inhalt und Form des Staates eine Einheit:

Im Mittelalter gab es Leibeigene, Feudalgut, Gewerbe-Corpartion, GelehrtenCorporation etc.; d.h. im Mittelalter ist Eigenthum, Handel, Societät, Mensch

\footnotetext{
14 Vgl. MEW I, S.232-233 / MEGA I/2, S.32-33.

15 MEW I, S.232 / MEGA I/2, S.32.

16 Ebd.
}

\begin{tabular}{|l|l|l|l|l|}
\hline Revista Dialectus & Ano 9 & n. 18 & Outubro 2020 & p. 275-296 \\
\hline
\end{tabular}


politisch; der materielle Inhalt des Staats ist durch seine Form gesetzt. (MEW 1, 233)

Dadurch, dass jede Privatsphäre auch politischen Charakter besitzt und damit alle Bereiche des Sozialen zugleich auch im Bereich des Politischen liegen, ist im Mittelalter „Volksleben und Staatsleben identisch.“ (MEW 1, 233) Allerdings folgert Marx daraus weder ein „Zurückins-Mittelalter“, noch dass diese integrative Sozialform ein Ideal darstellen würde, wie Teile seiner romantischen Zeitgenossen. Ganz im Gegenteil: zwar konstituiert das Politische eine soziale Einheit, indem es in alle sozialen Bereiche ausstrahlt und damit den ganzen, wirklichen Menschen zum Prinzip des Staates macht, gleichwohl ist es hier der „unfreie Mensch“ (ebd.), welcher das Prinzip des Staates ausmacht. Was Marx hier als politischen Charakter aller Bereiche des Sozialen fasst, ist politisch im Sinne personaler Herrschaft. Dementsprechend konstituiert sich die soziale Einheit des Mittelalters politisch als konkreter Gegensatz zwischen Herrschern und Beherrschten, während die soziale Trennung der Moderne einen Gegensatz zwischen wesenslogisch unterschiedlichen sozialen Sphären beschreibt. Marx schreibt: „Das Mittelalter ist der wirkliche, die moderne Zeit ist abstrakter Dualismus.“(ebd.)

Am Beispiel dieses Gegensatzes von Mittelalter und Moderne lässt sich die spezifische Marxsche Entwicklungslogik des Politischen und damit zugleich auch die Geschichte der Freiheit aufzeigen. Ihr politischer und freiheitstheoretischer Unterschied besteht darin, dass sich aus der sozialen Einheit des Mittelalters der politische Staat als eigene, genuin politische Sphäre herauslöste und zugleich die Sphäre des Privaten als genuin unpolitische konsolidierte. Anders als Hegel, fasst Marx diese historische Entwicklung der Moderne als Zäsur. Hegel argumentiert, dass durch die allmähliche Herausbildung des Verfassungsstaats, die sich fast unmerklich vollzog, die soziale Einheit durch die Verfassung - sprich dem politischen Staat erst eine Wirklichkeit erhielt. Diesem Narrativ widerspricht Marx in doppelter Hinsicht und seine Ausführungen dazu verdeutlichen in pointierte Weise seine anarchistische Position.

Erstens konstatiert Marx, dass diese sukzessiven politischen Veränderungen, von denen Hegel als konstitutive Verfassungselementen spricht (vgl. HGPR, 247), immer nur partikularen Charakters waren und nie eine qualitative Veränderung der sozialen und politischen Verhältnisse bedeuteten. Hegels Annahme sei schlichtweg „historisch falsch“ (MEW 1, 259). Marx schreibt:

\begin{tabular}{|l|l|l|l|l|}
\hline Q Rovista Dialectus & Ano 9 & n. 18 & Outubro 2020 & p. 275-296 \\
\hline
\end{tabular}


Ganze Staatsverfassungen haben sich allerdings so verändert, daß nach und nach neue Bedürfnisse entstanden, daß das Alte zerfiel etc.; aber zu der neuen Verfassung hat es immer einer förmlichen Revolution bedurft. (MEW 1, 259)

Marx' empirisch-essentialistischer Kritikmodus, welcher hier gegen Hegel in Anschlag gebracht wird, impliziert, dass diese Revolutionen immer ein politisches Handeln des Volkes sind. Auf Grundlage der Feuerbach'schen Inversion, zeigt Marx, dass sich nicht die Verfassung verändert, sondern in den Revolutionen das Volk selbst, ,also der wirkliche Träger der Verfassung“ (MEW 1, 259), die Verfassung macht. ${ }^{17}$ Dies ist, wie weiter unten noch ausgeführt wird, eine typisch anarchistische Argumentationsfigur.

Zweitens entgegnet Marx Hegels Argumentation, dass die bisherigen Verfassungen mitnichten die Objektivierung einer sozialen Einheit bilden, sondern gerade ihre Trennung vollziehen. Die politische Verfassung bildete sich als besonderes Element neben den anderen sozialen Sphären heraus, „als ein Jenseitiges derselben.“ (MEW 1, 232) Der Charakter des Verhältnisses dieser Trennung von politischem Staate und bürgerlicher Gesellschaft fußt auf Marx' Wesenslogik. Der politische Staat, welcher auf der Verfassung ruht, hat das Gemeinwohl, oder philosophisch gesprochen das Allgemeine oder den Gattungsinhalt zu seinem Gegenstand. Er stellt den Ort der allgemeinen Vernunft dar. Allerdings steht dem politischen Staat die bürgerliche Gesellschaft entgegen, deren Inhalt er gerade nicht bestimmt, da ihr Inhalt unpolitisch und bloße Privatsache ist. Da sich Privatleben und Gemeinwohl nicht politisch durchdringen, verbleibt die Verfassung, als das seinem eigenen Anspruch nach Allgemeine, in einem abstrakten Modus und bildet einen Gegensatz zum Privaten (vgl. (MEW 1, 233).

Aus Marx' empirisch-essentialistischem Ansatz ergibt sich, dass der Staat ein Resultat der Revolutionen des Volkes ist, aber dennoch seinen eigenen Anspruch - der im politischen Charakter der Revolution des Volkes selbst liegt, Vollstrecker des Allgemeinen zu sein verfehlt. Der Staat bleibt als bloß politischer so nur „ein Monopol“ der Politiker und Bürokraten, während die „Monopole“ in der bürgerlichen Gesellschaft „die wirklichen allgemeinen Angelegenheiten sind.“ (MEW 1, 268) Dennoch knüpft Marx nicht an einen

17 Marx knüpft hier an Feuerbachs politische Philosophie aus den Vorläufige Thesen zur Reform der Philosophie an, in denen die Idee entwickelt wird, dass der Staat ,die realisierte, ausgebildete,

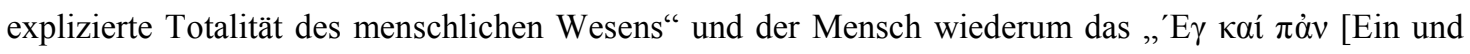
alles, S.W.] des Staates“ ist. (Feuerbach 1970, 262)

\begin{tabular}{|l|l|l|l|l|}
\hline Q Ponista Dialectus & Ano 9 & n. 18 & Outubro 2020 & p. 275-296 \\
\hline
\end{tabular}


etatistischen Geltungsüberschuss an, welcher reformistisch sich realisieren lassen würde, sondern spricht sich vielmehr für einen revolutionären Bruch aus.

Als eine notwendige Entwicklungsstufe bildet diese moderne Entfremdung des Sozialen, in Form der Trennung zwischen politischem Staat und unpolitischer bürgerlicher Gesellschaft, die Bedingung für die revolutionäre Verwirklichung des Gattungsinhalts durch eine angemessene Realisierung des Poltischen. Diese Versöhnung von Allgemeinen und Besonderem, von Subjekt und Objekt, sowie der Vereinheitlichung des Sozialen und des Politischen, konzeptualisiert Marx mit dem Begriff der „wahren Demokratie““. ${ }^{18}$ Während Hegel der Demokratie als unvernünftiges und unorganisches Extrem eines politischen Radikalismus deutlich eine Absage erteilt, nimmt sie in Marx' Sozialphilosophie genau jene zentrale Stellung ein, die Hegel dem Staat zuschreibt.

Marx und der Anarchismus

Im Folgenden werde ich nun den Marxschen Anarchismus explizit umreißen, wie er stellenweise immer wieder in der Kritik der Hegelschen Rechtsphilosophie in Anschlag gebracht wird. Was meines Erachtens den anarchistischen Entwurf Marx' in besonderer Weise auszeichnet, ist der Versuch einer weitgehenden Unvermitteltheit zwischen den politischen Subjekten und ihren politischen Objektivationen, wie der Verfassung, den Institutionen, usw.

Anders als in klassischen Demokratietheorien erhält die demokratische Verfassung bei Marx bloß einen zeitweiligen, temporären Status. Die Verfassung ist schließlich eine vorübergehende Objektivierung des jeweiligen politischen Willens der Menschen. Die Verfassung wird dementsprechend immer wieder neu, den Bedürfnissen und dem Willen der Menschen entsprechend konstituiert und bildet damit die objektivierte Seite der (fast) unvermittelten Selbstherrschaft des Volkes. Während in allen bisherigen politischen Systemen die Objektivierungen des Menschen über die Menschen herrschten, so soll in der wahren Demokratie das Verhältnis umgedreht werden und die Verfassung unterliegt dem Willen des Volkes. In der wahren Demokratie „erscheint die Verfassung selbst nur als eine Bestimmung, und zwar Selbstbestimmung des Volks.“ (MEW 1, 231) Exakt in dieser stetigen

18 Marx Demokratiekonzeption reicht in ihrem demokratischen Anspruch deutlich weiter als jegliche zeitgenössische Demokratie, wie bspw. in Nordamerika. Aber auch theoretisch vertritt er hier einen radikal-demokratischen Ansatz, der nichts mit liberalen Demokratietheorien gemein hat (vgl. Avineri 1969, 33-34; sowie MEW 1, S.230f.).

\begin{tabular}{|l|c|c|c|c|}
\hline Q Rovista Dialectus & Ano 9 & n. 18 & Outubro 2020 & p. 275-296 \\
\hline
\end{tabular}


basisdemokratischen Aktualisierung der Verfassung durch das Volk - und damit des gesamten politischen Systems - liegt Marx’ Anarchismus.

Damit schafft Marx in seiner Konzeption den klassischen Staat ab. Da der Staat einerseits Ausdruck der Trennung ist und andererseits diese Trennung aktiv vollzieht, soll er als eigenständige Institution durch eine Revolution aufgehoben werden. Er ist schlichtweg nicht notwendig, da das Volk als wesenslogisches Fundament des Politischen in der wahren Demokratie sich selbst regiert - daher sagt Marx der Demokratie nach, sie sei „,das aufgelöste Räthsel aller Verfassungen.“ (MEW 1, 230)

Doch zugleich bleibt eine Form des Staates erhalten, die allerdings mit den bisherigen Staatsformen nichts gemein haben kann. Marx mach deutlich, dass in der wahren Demokratie „keines der Momente eine andere Bedeutung“ erlangt, „als ihm zukommt. Jedes ist wirklich nur Moment des ganzen Demos.“ (MEW 1, 230) Dies gilt auch für den Staat. Marx schreibt an keiner Stelle von der Abschaffung des Staates schlechthin, sondern allein von der Aufhebung der Trennung von Form und Inhalt des Staates, von der Überwindung des Dualismus zwischen abstraktem und materiellem Staat und von dem Ende der politischen Entfremdung. Wird das Verhältnis zwischen Staat und Volk in gleicher Weise wie in Bezug auf die Verfassung umgekehrt, so wird deutlich, dass auch der Staat eine Einhegung seiner Kompetenzen, seines Geltungsbereichs und schlussendlich das Ende seiner Autorität erfährt (vgl. MEW 1, 230f.). Gleichwohl scheint eine Art Reststaat in der Marxschen Konzeption bestehen zu bleiben, den man allerdings eher als eine Art Ausschuss begreifen müsste - Marx Ausführungen dazu sind sehr vage. Jedenfalls sollen sich weder Verfassung noch Staat in der wahren Demokratie verdinglichen können. Als verflüssigte Resultate des freien Gattungswillens sind sie zugleich Produkte des fortwährenden politischen Schaffens (vgl. Abensour 2012, 144-147).

Ferner schließt die Aufhebung der Trennung zwischen Staat und bürgerlicher Gesellschaft die Aufhebung des Privateigentums, der Klassen und des Prinzips der bürgerlichen Gesellschaft der Individualismus - mit ein. Indes verbleiben Marx Ausführungen auch dazu oberflächlich. ${ }^{19}$ Avineri interpretiert die synchrone Aufhebung des politischen Staates und der

19 So geht Marx auf die Aufhebung der bürgerlichen Gesellschaft und des Privateigentums nur selten ein und schon gar nicht auf das Verhältnis der verschiedenen Klassen zueinander. Wie naiv Marx sich hier die Auflösung der bürgerlichen Gesellschaft vorstellt, zeigt sich unteranderem daran, dass er glaubt mit dem allgemein Wahlrecht die bürgerliche Gesellschaft zu überwinden: „Die Wahlreform ist also innerhalb des abstrakten politischen Staats die Forderung seiner Auflösung, aber ebenso der Auflösung

\begin{tabular}{|c|c|c|c|c|}
\hline Rovista Dialectus & Ano 9 & n. 18 & Outubro 2020 & p. 275-296 \\
\hline
\end{tabular}


bürgerlichen Gesellschaft als Vorform von Marx' späteren Kommunismus, da aus Marx' Kritik und seinen Prämissen notwendig die Abschaffung des Privateigentums folgt (vgl. Avineri 1969, 34-38). Allerdings bleibt durchaus fraglich, was Marx unter Privateigentum überhaupt versteht, welche Verkehrsformen er im Blick hat und schließlich fehlt dem Manuskript jeglicher Klassenbezug. Demgegenüber scheint es angebrachter von dem Primat der Politik zu sprechen, weshalb es treffender ist, das Programm der wahren Demokratie als ein anarchistisches zu verstehen. ${ }^{20}$

Es sprechen aber auch eine Reihe von strukturellen Gründen dafür, das Programm der wahren Demokratie als eine anarchistische Konzeption zu lesen sprechen. Dies lässt sich an den strukturellen Affinitäten von Marx' Hegelkritik zu dem Anarchismus des 19. und frühen 20. Jahrhunderts aufzeigen.

Zunächst wird dies an dem Stellenwert der Anthropologie deutlich. Typisch für den Anarchismus des 19. und frühen 20. Jahrhundert ist die Fundierung des Politischen in der Anthropologie, so wie man sie bei Bakunin (2013), Büchner (1906) oder Kropotkin (1923) finden kann. Exakt ein solches Fundierungs-Paradigma des Politischen liegt Marx' essentialistisch-empirischen Ansatz zugrunde. Seine Methode der Dekonstruktion des Sittlichen besteht schlussendlich in der Rückführung derselben in eine politische Anthropologie des Gattungswesens. Diese radikale Rückführung der Hegelschen Kategorie auf ein Subjekt, d.h. dem Menschen als Wesen der Wesen, führt dazu „die Tätigkeit“ aus Hegels Geistphilosophie zu verbannen und „auf ein gegenständliches Substrat“ aufzutragen (Theunissen 1978, 483). D.h., dass sämtliche Kategorien des Politischen und Sozialen von Hegels Ideen weg in einem Fluchtpunkt des Gemeinsamen enggeführt werden, den Marx mal den „Menschen“, ein anderes Mal die „Gattung“ oder das „Volk“ nennt. ${ }^{21}$

Wenn Marx von der Realisierung des Gattungswesens spricht, so meint er 1843 damit die Aufhebung der politischen Gegensätze zwischen individuellem und allgemeinem Menschen. Die politischen Objektivierungen des Menschen, die entstehen, wenn er sich sein eigenes Wesen zum Gegenstand, zum Objekt macht, waren bisher aufgrund ihres verselbständigten verdinglichten Charakters entfremdete Gemeinwesen und Staaten. Dementsprechend

der bürgerlichen Gesellschaft“ (MEW 1, S.326-327). Andere Stellen dazu finden sich auf: MEW 1, S.232, 253, 303 .

20 Eine ähnliche Interpretation findet sich auch bei Loick 2010, 157.

21 Mensch und Gattung werden bei Marx synonym verwendet, während meiner Ansicht nach das „Volk“ der dezidiert politische Modus des Menschen ist.

\begin{tabular}{|l|l|l|l|l|}
\hline Q Rovista Dialectus & Ano 9 & n. 18 & Outubro 2020 & p. 275-296 \\
\hline
\end{tabular}


beschreibt die wahre Demokratie als der politische Ausdruck des Gattungswesen kein organisches System der Vermittlung wie bei Hegel, sondern eine sich stetig selbst aktualisierende Unmittelbarkeit. Sie ist, wie Lindner schreibt, „ein Gestaltungsmodus des Sozialen, in dem die politische Gestaltungsform nicht mehr über, sondern in dem ZuGestaltenden liegt.“ (Lindner 2013, 87) Form und Inhalt unterliegen zwar auch in der wahren Demokratie der Dialektik der Entfremdung und Objektivierung, aber da die Verfassung, der Staat, etc. wesentlich auf das Volk rückbezogen sind, bilden sie in dieser sich aktualisierenden Immanenz immer wieder eine Identität.

Eine weitere starke Affinität von Marx' Hegelkritik zum Anarchismus besteht in der Rückführung des Politischen von Hegels Kategorien des Geistes und der Idee auf das Handeln der Menschen. Der damit einhergehende Voluntarismus ist ebenfalls ein typisches Strukturelement des Anarchismus. Indem damit Geschichte als ein Kontinuum menschlichen Handelns erschlossen wird, werden die Verhältnisse wandelbar und damit soziale Ordnung durch den Menschen machbar. Mit dem Politischen geht es aber Marx nicht primär um Fragen der Herrschaft, sondern, wie Abensour schreibt, vielmehr darum, „wie das menschliche Zusammenleben $\mathrm{zu}$ gestalten ist, damit es den Anforderungen der Freiheit"“ entspricht (Abensour 2012, 127). Da sich die Frage der Gestaltung des Gemeinwesens an den politischen und handelnden Menschen richtet, wird deutlich, dass Marx' Konzeption von 1843 zwingend voluntaristische Züge trägt. Es gilt sozusagen angesichts der Fragmentierung und Zersplitterung des Gattungswesens eine Einheit $\mathrm{zu}$ schaffen. Ein freiheitliches Zusammenleben kann nach Marx nur Resultat einer Tat aus Freiheit sein (vgl. Avineri 1969, 32f.). Die Nähe zu Bakunin ist auch hier mehr als deutlich. Schon ein Jahr zuvor proklamierte Bakunin, dass die Frage der Freiheit „keineswegs durch eine formale Anwendung und Verbreitung von fertigen Theorien“ beantwortet werden könne, „sondern nur durch eine ursprüngliche Tat des praktischen autonomischen Geistes“ vollbracht wird (Bakunin 1984, 32). Die anthropologische Wende impliziert also zugleich eine prometheische Praxis der Freiheit.

Und schließlich untersteht Marx' Konzeption von 1843 einem typischen anarchistischen Primat des Politischen. Auch wenn sich Marx durchaus des Problemkomplexes der bürgerlichen Gesellschaft und ihren Strukturen bewusst ist, werden diese 1843 (noch) nicht explizit behandelt. Das Zentrum der Kritik und des Gegenentwurfs stellt die Aufhebung der politischen Entfremdung dar, weshalb Avineris oben genannte These, dass hier schon der

\begin{tabular}{|l|l|l|l|l|}
\hline Qevista Dialectus & Ano 9 & n. 18 & Outubro 2020 & p. 275-296 \\
\hline
\end{tabular}


Kommunismus aufscheine, nicht plausibel ist. Marx' Staatskritik zielt darauf ab, das Politische zum Gegenstand des Wesens des Politischen, des Menschen zu machen. Indem in der wahren Demokratie dezidiert die politischen Vermittlungsinstanzen auf ein Mindestmaß beschränkt und zugleich immer dem Willen des Volkes unterstellt sein sollen, wird der Staat als solcher aufgehoben. ${ }^{22}$ Diese autopoetische Konzeption des politischen Menschen entspricht exakt jener Definition des Anarchismus, die Peter Kropotkin 68 Jahre später in der Encyclopedia Britannica geben wird:

\begin{abstract}
Anarchism $[\ldots]$, the name given to a principle or theory of life and conduct under which society is conceived without government - harmony in such a society being obtained, not by submission to law, or by obedience to any authority, but by free agreements concluded between the various groups, territorial and professional, freely constituted for the sake of production and consumption, as also for the satisfaction of the infinite variety of needs and aspirations of a civilized being. (Kropotkin 1911, 914)
\end{abstract}

Der Anarchismus Marx' verbleibt als bloße Skizzierung eines nie dagewesen Zustands allerdings noch in der Sphäre der Idealität. 1843 ist noch nichts von dem „Kommunismus“ als der ,wirkliche[n] Bewegung, welche den jetzigen Zustand aufhebt“ (MEW 3, 35) zu lesen. Der Anarchismus stellt für Marx vielmehr eine regulative Idee dar, mit der sowohl die Vergangenheit verstanden als auch die Gegenwart gedeutet werden kann. Damit ist Marx auf einer Linie mit seinem späteren Konkurrenten Pierre-Joseph Proudhon. Obzwar Proudhon Marx' Konzeption des Volkswillens abgelehnt hätte, so treffen sich beide in dem bestimmenden Ideal einer auf Selbstherrschaft des Menschen gründenden möglichst unvermittelten Selbstorganisation der Gesellschaft. Entgegen seiner nur fünf Jahre späteren Geringschätzung des Anarchisten, hätte Marx 1843 den folgenden Ausführungen Proudhons nur lobend zustimmen können:

Es hat niemals das Beispiel einer vollkommenen Gemeinherrschaft gegeben, und es ist recht unwahrscheinlich, daß alle Spuren der Regierung und der Autorität verschwinden werden, auch wenn die Menschheit einen noch so hohen Stand der Zivilisation, Sittlichkeit und Weisheit erreicht. Doch ist [...] die Anarchie das Ideal [...] das darauf drängt, jede Verfestigung der Regierung abzuschaffen [...]. (Proudhon 1963, 208f.)

22 Auch hier besteht eine deutliche Nähe zu seinem Zeitgenossen Bakunin (vgl. Bakunin 2013, 89).

\begin{tabular}{|c|c|c|c|c|}
\hline Rovista Dialectus & Ano 9 & n. 18 & Outubro 2020 & p. $275-296$ \\
\hline
\end{tabular}


Resümee

Angesichts dieser Nähe des frühen Marx zum Anarchismus ist es durchaus angebracht in Anlehnung an Johann Most die späteren Konflikte Marx“ mit Proudhon und besonders mit Bakunin als einen „Bruderzwist“ zu bezeichnen (Most 2006, 108). Gleichwohl treffen Marx mit dieser Nähe zum Anarchismus auch jene Kritiken, die auch für anarchistische Theorien zutreffen. ${ }^{23}$ Wie auch in der Kritik des Anarchismus immer wieder deutlich gemacht wurde, so besteht meines Erachtens die größte Problematik von Marx' Anarchismus in seinem Volksbegriff. Da das Volk als politischer Ausdruck des Gattungswesens eine Identität bildet, finden Konflikte allein zwischen der stetigen Aktualisierung des Volkswillens mit seinen Objektivierungen, wie der Verfassung, dem Staat, etc. statt, werden allerdings aufgrund der Identität von Form und Inhalt immer wieder aufgehoben. Eine Konflikthaftigkeit im Volk, d.h. im politischen Gattungswesen selbst scheint es für den Marx von 1843 nicht zu geben (vgl. Lindner 2013, 88). Als eine solche identätslogische, positive Grundkategorie beschreibt das Volk die vollkommene soziale Einheit und Auflösung der Widersprüche des Sozialen in einer politischen Gemeinschaft der Menschen.

Wie bereits ausgeführt, wendet Marx die typisch linkshegelianische und im Besonderen Feuerbachsche Figur der Rückführung idealistischer Kategorien auf das Soziale, auf den Bereich des Politischen an. Das Volk manifestiert 1843 seinen Kollektivwillen in der Demokratie in Form der Souveränität, in seiner Selbstherrschaft. Es ist die schon immer dagewesene politische Substanz, welche sich in der Demokratie die entsprechende Form der Objektivierung geben kann. Nicht die Demokratie als dezentrale partizipative politische Form konstituiert ein Volk der Demokratie, sondern vice versa: das Volk konstituiert die Demokratie, obwohl es sich beim Volk, wie Demirovic richtig anmerkt, „de facto um eine Reflexionskategorie handelt.“ (Demirović 1997, 62)

Gleichwohl bleibt bei Marx offen, wer oder was dieses Volk sei. Außerhalb oder unterhalb der sozialen Existenzweisen der Familie, der bürgerlichen Gesellschaft und des Staates stehend, ist das Volk das homogene politische Kollektivsubjekt.

Ironischerweise abstrahiert Marx hier gerade von jenen sozialen Differenzierungen, die er bei Hegel lobt, und konstruiert eine Homogenität, die trotz des Individualismus der bürgerlichen Gesellschaft, sowie der generellen Trennung zwischen Citoyen und Bourgeois, besteht. In

23 Zur Kritik des klassischen Anarchismus vgl. Newman 2010, 393.

\begin{tabular}{|l|l|l|l|l|}
\hline Ronista Dialectus & Ano 9 & n. 18 & Outubro 2020 & p. 275-296 \\
\hline
\end{tabular}


völligem Gegensatz zu seinem historisch-empirischen Ansatz, die Wirklichkeit des Staates, der Gesellschaft und der Geschichte zu analysieren, kittet Marx das Soziale durch eine leere, fiktionale politische Kategorie zusammen. ${ }^{24}$

Man kann sicher einwenden, dass der Impetus einer solchen Konzeption doch gerade in Herrschaftsfreiheit läge, jedoch zielt das anarchistische Element in Marx' wahrer Demokratie gerade nur auf die Vertikalität politischer Herrschaft und ignoriert damit jegliche Form horizontaler Gleichschaltung als Herrschaftsform. Diese Form der Versöhnung unterläuft sich genau darin selbst, dass sie der Artikulation eines Volkswillens bedarf und eine Homogenität notwendig macht. Man müsste mit Hegel polemisch an Marx zurückfragen: was ist mit den Einzelnen, in diesem „Alle“ des Volkes, also jenen einzelnen Individuen, deren vielfältige und unterschiedliche Interessenslagen und deren Autonomie einem solchen Kollektivwillen widersprechen?

Gleichwohl sieht Marx selbst, dass er sich in diesen Aporien verfängt, welche sich allerdings aus der Sache selbst ergeben. Deshalb wendet er sich ab 1844 von der politischen Theorie und Kritik ab, hin zur Kritik der politischen Ökonomie. Dennoch wird das anarchistische Moment von 1843 immer den Fluchtpunkt für eine emanzipatorische Form des Politischen bilden. ${ }^{25}$

\section{Siglen}

HGPR: Hegel, Georg Wilhelm Friedrich: Grundlinien der Philosophie des Rechts. In: Georg Wilhelm Friedrich Hegel: Hauptwerke in sechs Bänden, Bd. 5. Hamburg: Meiner, 2015.

MEGA: Marx, Karl; Engels, Friedrich: Gesamtausgabe. Berlin/ Boston: De Gruyter, 1975.

24 Selbstverständlich ist dieser Übergang von einem materialistischen Anspruch in eine politische Identitätslogik eine Wiederholung und politische Exemplifizierung der ahistorischen und anthropologischen Seite des Gattungswesens Feuerbachs. Die Anthropologisierung des Idealismus gebiert dementsprechend einen neuen Mystizismus, was Marx selbst einige Jahre später selbst feststellen wird.

25 Zu nennen wäre hier beispielsweise die Kritik der Repräsentation, welche Marx fast exakt so wie 1843 noch 1871 in Der Bürgerkrieg in Frankreich wiederholt (vgl. MEW 17, S.339-342). Aber auch Marx selbst betont die eigene Schaffenskontinuität im Vorwort Zur Kritik der politischen Ökonomie von 1859: „Die erste Arbeit, unternommen zur Lösung der Zweifel, die mich bestürmten, war eine kritische Revision der Hegelschen Rechtsphilosophie, eine Arbeit, wovon die Einleitung in den 1844 in Paris herausgegebenen ,Deutsch-Französischen Jahrbüchern' erschien. Meine Untersuchung mündete in dem Ergebnis, daß Rechtsverhältnisse wie Staatsformen weder aus sich selbst zu begreifen sind noch aus der sogenannten allgemeinen Entwicklung des menschlichen Geistes, sondern vielmehr in den materiellen Lebensverhältnissen wurzeln, deren Gesamtheit Hegel, nach dem Vorgang der Engländer und Franzosen des 18. Jahrhunderts, unter dem Namen "bürgerliche Gesellschaft" zusammenfaßt, daß aber die Anatomie der bürgerlichen Gesellschaft in der politischen Ökonomie zu suchen sei“" (MEW 13, S.8).

\begin{tabular}{|l|l|l|l|l|}
\hline Revista Dialectus & Ano 9 & n. 18 & Outubro 2020 & p. 275-296 \\
\hline
\end{tabular}


MEW: Marx, Karl; Engels, Friedrich: Werke. Berlin: Dietz, 1975.

\section{Literatur}

Abensour, Miguel: Demokratie gegen den Staat. Marx und der machiavellische Moment. Berlin: Suhrkamp, 2012.

Althusser, Louis: Für Marx. Frankfurt/M.: Suhrkamp, 1968.

Avineri, Shlomo: The social and political thought of Karl Marx. London: Cambridge University Press, 1969.

Bakunin, Michael Aleksandrovič: Gott und der Staat (Ausgewählte Schriften, Bd. 1. Hrsg. von Wolfgang Eckhardt; Bd. 1). Berlin: Kramer, 2013.

Bakunin, Michail Aleksandrovič: Die Reaktion in Deutschland. Hamburg: Nautilus, 1984.

Büchner, Ludwig: Darwinismus und Sozialismus oder der Kampf um das Dasein und die moderne Gesellschaft. Stuttgart: Kröner, 1906.

Demirović, Alex: Demokratie und Herrschaft. Aspekte kritischer Gesellschaftstheorie. Münster: Westfälisches Dampfboot, 1997.

Feuerbach, Ludwig: Vorläufige Thesen zur Reformation der Philosophie. In: Ludwig Feuerbach: Gesammelte Werke. Kleinere Schriften II (1839-1846). Hrsg. v. Werner Schuffenhauer. Berlin: Akademie Verlag, 1970, S. 243-263.

Hegel, Georg Wilhelm Friedrich: Grundlinien der Philosophie des Rechts. In: Georg Wilhelm Friedrich Hegel: Hauptwerke. In sechs Bänden; Bd. 5. Hamburg: Meiner, 2015.

Hoffmeister, Johannes: Dokumente zu Hegels Entwicklung. Stuttgart: Frommann, 1936.

Hyppolite, Jean: Studies on Marx and Hegel. London: Heinemann, 1969.

Kropotkin, Pëtr Alekseevič: Gegenseitige Hilfe in der Tier- und Menschenwelt. Leipzig: Thomas, 1932.

Leopold, David: The young Karl Marx. German philosophy, modern politics, and human flourishing. Cambridge: Cambridge Univinversity Press, 2009.

Lindner, Urs: Marx und die Philosophie. Wissenschaftlicher Realismus, ethischer Perfektionismus und kritische Sozialtheorie. Stuttgart: Schmetterling, 2013.

Loick, Daniel: Kritik der Souveränität. Frankfurt: Campus, 2012.

Most, Johann: Der kommunistische Anarchismus. In: Johann Most: Die freie Gesellschaft. Münster: Unrast, 2006, S. 95-122.

\begin{tabular}{|c|c|c|c|c|}
\hline Rovista Dialeatus & Ano 9 & n. 18 & Outubro 2020 & p. $275-296$ \\
\hline
\end{tabular}


Newman, Saul: From Bakunin to Lacan. Anti-authoritarianism and the dislocation of power. Lanham, Md: Lexington Books, 2010.

Proudhon, Pierre-Joseph: Über das föderative Prinzip und die Notwendigkeit, die Partei der Revolution wiederherzustellen. In: Pierre-Joseph Proudhon: Ausgewählte Texte. Hrsg. V. Thilo Ramm. Stuttgart: K.F. Koehler Verlag, 1963, S. 193-264.

Riedel, Manfred: Bürgerliche Gesellschaft. Eine Kategorie der klassischen Politik und des modernen Naturrechts. Stuttgart: Steiner, 2011.

Ritter, Joachim: Hegel und die Französische Revolution. Köln: Westdeutscher Verlag, 1957.

Rubel, Maximilien: Marx-Chronik. Daten zu Leben und Werk. München: Hanser, 1968.

Theunissen, Michael: Sein und Schein. Die kritische Funktion der Hegelschen Logik. Frankfurt a.M.: Suhrkamp, 1978.

\begin{tabular}{|l|l|l|l|l|}
\hline Q Rovista Dialectus & Ano 9 & n. 18 & Outubro 2020 & p. 275-296 \\
\hline
\end{tabular}

